\title{
A new approach for solving dual-hesitant fuzzy transportation problem with restrictions
}

\author{
GURUPADA MAITY $^{1}$, DHARMADAS MARDANYA ${ }^{2}$, SANKAR KUMAR ROY ${ }^{1, *}$ and \\ GERHARD-WILHELM WEBER ${ }^{3}$ \\ ${ }^{1}$ Department of Applied Mathematics with Oceanology and Computer Programming, Vidyasagar University, \\ Midnapore 721102, India \\ ${ }^{2}$ Department of Mathematics, Sai Sadhana College of Basic Sciences, Mayurbhanj 757041, India \\ ${ }^{3}$ Department of Engineering Management, Poznan University of Technology, Poznan, Poland \\ e-mail: sankroy2006@gmail.com
}

MS received 28 February 2018; revised 7 September 2018; accepted 12 October 2018; published online 11 March 2019

\begin{abstract}
This paper addresses a study on the transportation problem based on dual-hesitant fuzzy environment. The dual-hesitant fuzzy set accommodates imprecise, uncertain or incomplete information and knowledge situations in real-life operational research problems that are not possible or difficult to tackle by existing fuzzy uncertainties. Here, we present the concept of uncertainty in a transportation problem using dual-hesitant fuzzy numbers. In most of the research works, fuzzy uncertainty has been considered in transportation parameters. However, we consider the dual-hesitant fuzzy numbers to formulate a mathematical model by considering the capacity of delivering the goods by a decision maker. A special emphasis of this paper is to derive an optimal solution of transportation problem with some restrictions under uncertainty by the traditional approach (cf. Vogel's approximation method-VAM) without using any mathematical aids. In this regard, an algorithm is developed to find the optimal solution for the dual-hesitant fuzzy transportation problem including some restrictions. Thereafter, the proposed method is illustrated by giving a numerical example for showing the effectiveness. Finally, conclusions are given with the lines of future studies based on this paper.
\end{abstract}

Keyword. Transportation problem; fuzzy sets; dual-hesitant fuzzy sets; uncertainty; score function.

\section{Introduction}

At the beginning of the 21st century, transportation professionals meet challenges to address the increasing complexity. Transportation professionals are asked to achieve the goals for providing efficient, safe and reliable transportation while minimizing the impact on the environment and communities. A partial listing of those challenges that transportation professionals face includes capacity restrictions, environmental pollution, poor safety record, unreliability and wasted energy. Considering these facts, transportation systems are generally complex systems involving a large number of components and different parties, each having different and often conflicting objectives.

Transportation Problem (TP) is mainly considered to minimize the transportation cost from sources to numerous destinations at an early time of study in Operations Research (OR). In our real-life situations, several decisionmaking problems such as fixing the cost of goods, profit for

*For correspondence sellers, taking decisions for real-life multiple objective functions, etc. are solved by the TP. Nowadays, in the competitive market scenario, minimizing the transportation cost in a business economy and in government policies is one of the utmost important challenges.

The basic TP was originally developed by Hitchcock [1] and later on advanced independently by Koopmans [2] and Kantorovich [3]. A number of research works have been carried out by various authors in different areas of TP, such as those by Chanas et al [4], Mahapatra et al [5], Maity and Roy [6], Maity et al [7], Maiyar and Thakkar [8], Mogale et al [9], Roy and Maity [10], Rani and Gulati [11], Roy et al $[12,13]$ and numerous others. Realizing the actual phenomena of TP, in most of the research works on TP, the authors minimized the total transportation cost using different approaches, and they established their utilities showing different approaches for solving TP using various kinds of data from different grounds. In almost all of the existing studies, no one shows interest on formulating a model of TP where the minimization of transportation cost is not only the main issue, but also the possibility of transportation to the right destinations in prescribed time is an utmost important factor. Here, we attempt to formulate a 
Dual-Hesitant Fuzzy Transportation Problem (DHFTP) in which the main aim is to minimize transportation cost with some restrictions of transporting goods to the destinations.

Nowadays, many real-life decision-making problems belong to the category "under uncertainty". In this regard, a study of the TP under fuzziness is valuable for tackling complex decision-making problems, whenever the information about a TP is only partially known. A fuzzy TP is a task of OR where the transportation cost, supply and demand quantities are taken as fuzzy numbers. Several existing methods are available to provide crisp solutions only for a fuzzy TP. Chanas and Kuchta [14] presented a procedure for solving a fuzzy TP. Kaur and Kumar [15] introduced a technique for solving a TP in a fuzzy environment, assuming some ambiguity of transportation cost, availability and demand parameters. Jiménez and Verdegay [16] studied solid TP in fuzzy environment and presented evolutionary algorithms to solve it. Recently, Roy et al [17] presented a new approach for solving intuitionistic fuzzy multi-objective TP. In the aforementioned research works (cf. [14-17]), fuzzy uncertainties are considered in transportation parameters but the mathematical models are not developed using these uncertain parameters. They do not provide enough information about the reason for using fuzzy criterion in the models. Moreover, there are a large number of studies available for solving TP under the assumption of fuzziness in at least one transportation parameter, but the field of study in which uncertainty affects real-life situations is not properly discussed. There may occur situations in time of transporting goods when vehicles through schedule routes are not sufficient enough in the hand of a decision maker (DM); hence, either he would have to decline the delivery or delay delivery of items to destinies. In this case, scheduling a supplementary way for delivering goods is required, paying more cost than the optimum cost of transportation. As a result, the transportation cost considered in the TP does not remain fixed, which is also not possible to tackle by traditional fuzzy cost parameter. To recover the difficulty in practical situations, we incorporate a new class of fuzziness, namely, dual-hesitant fuzzy number, which accommodates the situation nicely and provides a better result in comparison with existing studies under fuzziness.

Torra and Narukawa [18] first initiated the concept of a Hesitant Fuzzy Set (HFS). Torra [19] gave a definition corresponding to the envelope of a HFS. Zhu et al [20] proposed Dual-Hesitant Fuzzy Sets (DHFSs), which are an extension of HFSs that encompass fuzzy sets, intuitionistic fuzzy sets, HFSs and fuzzy multi-sets as special cases. Torra [19] and Zhu et al [20] introduced the basic properties and operations of DHFSs. Thereafter, they presented the concept of DHFSs in a group forecasting problem. A study on solving assignment problems with DHFSs was presented by Singh [21]. A deficiency in his paper is that the occurrence of that uncertainty is not described in detail. Furthermore, the assignment problem is a special case of the TP although the author [21] has not attempted to solve TP. Hence, we formulate DHFTP based on reality. Maity and Roy [22] studied TP using a fuzzy decision variable in multi-objective environment. In their study, the authors considered the decision variable as fuzzy numbers, as there was not enough information on hesitant fuzzy numbers for formulating the model. Basically, there are two parts of a DHFS; one is given by hesitancy membership function, and the other one by hesitancy non-membership function. One may also realize that existing sets such as fuzzy sets, intuitionistic fuzzy sets, HFSs and fuzzy multi-sets can be considered as special cases of DHFSs. The degrees of membership and non-membership functions of DHFSs are regarded as two sets of possible values. Therefore, DHFS has expected characteristics and its own advantages; it appears to be a more suitable method, valued in multi-fold ways according to practical demands, taking into account much more information given to the DMs.

The contributions of various researchers in this field are presented in table 1 .

Nowadays, most research works are carried out by means of mathematical aids; basically, the problems are solved through the use of a software (cf. [9, 23-25]). However, sometimes we observe that when the solution corresponding to a problem is possibly obtained through classical approaches without using mathematical aids, then it is treated as more effective in research. In this paper, we introduce algorithms to find the optimal solution of our considered model; after this, the classical approach VAM is used to solve the problem without mathematical software. In addition to this, we also show that our approach can solve existing research problems through some counterexamples. In this regard, our paper is different from the existing research works.

In this work, we formulate the model of TP by considering the dual-hesitant fuzzy numbers. We state that the following are the main contributions of our paper:

- Designing a new class of TP, namely, DHFTP.

- Optimizing transportation cost based on the vehicle restrictions through a single objective optimization problem.

- DHFTP is solved using VAM without using any mathematical aids.

- The feasibility of obtained results is tested and compared to those of the existing methods.

The rest of the paper is organized as follows: In section 2, we introduce the problem environment of our study. Preliminaries to fuzzy sets and DHFs are presented in section 3. Section 4 presents the formulation of mathematica model. The solution procedure of Dual Hesitant Fuzzy Transportation Problem with Restrictions (DHFTPR) is stated in section 5 . Then, in section 6 , we illustrate the usefulness of the proposed technique with a numerical example. In section 7, we compare our new contribution to 
Table 1. Contributions of different authors related to TP under uncertainty; TC: transportation cost.

\begin{tabular}{|c|c|c|c|c|c|c|}
\hline Author(s) & $\begin{array}{l}\text { Fuzzy cost } \\
\text { parameter }\end{array}$ & $\begin{array}{c}\text { Hesitant fuzzy } \\
\text { number }\end{array}$ & $\begin{array}{c}\text { Optimizing TC } \\
\text { without restriction }\end{array}$ & $\begin{array}{l}\text { Optimizing under } \\
\text { restriction }\end{array}$ & $\begin{array}{l}\text { Solved using } \\
\text { software }\end{array}$ & $\begin{array}{c}\text { Solved not using } \\
\text { software }\end{array}$ \\
\hline Koopmas (1949) & & & $\sqrt{ }$ & & & $\sqrt{ }$ \\
\hline Kantorovich (1960) & & & $\sqrt{ }$ & & & $\sqrt{ }$ \\
\hline Chanas et al (1984) & $\sqrt{ }$ & & $\sqrt{ }$ & & $\sqrt{ }$ & \\
\hline Chanas and Kuchta (1996) & $\sqrt{ }$ & & $\sqrt{ }$ & & $\sqrt{ }$ & \\
\hline Jimenez and Verdagey (1999) & $\sqrt{ }$ & & $\sqrt{ }$ & & $\sqrt{ }$ & \\
\hline Kaur and Kumar (2012) & $\sqrt{ }$ & & $\sqrt{ }$ & & $\sqrt{ }$ & \\
\hline Mahapatra et al (2013) & $\sqrt{ }$ & & $\sqrt{ }$ & & $\sqrt{ }$ & \\
\hline Maity et al (2016) & $\sqrt{ }$ & & $\sqrt{ }$ & & $\sqrt{ }$ & \\
\hline Rani and Gulati (2016) & $\sqrt{ }$ & & $\sqrt{ }$ & & $\sqrt{ }$ & \\
\hline Roy and Maity (2017) & $\sqrt{ }$ & & $\sqrt{ }$ & & $\sqrt{ }$ & \\
\hline Roy et al (2017) & $\sqrt{ }$ & & $\sqrt{ }$ & & $\sqrt{ }$ & \\
\hline Our paper & $\sqrt{ }$ & $\sqrt{ }$ & & $\sqrt{ }$ & & $\sqrt{ }$ \\
\hline
\end{tabular}

other existing methods. Eventually, a conclusion of our new approach together with an outlook to future research is presented in section 8 .

\section{Problem environment}

A large number of studies have been addressed by various researchers for solving the TP in different uncertain environments. Nowadays, uncertainty of fuzziness of transportation parameters is considered for solving a real-life TP in numerous situations. Here, we are mainly interested to include various real phenomena for which the dual-hesitant fuzzy sense is incorporated into the proposed study of a TP.

Though the TP in its classical sense is formulated to find the minimum transportation cost, TP is not bound to its classical characteristics. Hesitant fuzzy numbers provide two membership functions for a single choice of the fuzzy cost parameter in the TP. The first membership function refers to the preference for selecting a crisp cost, whereas the second one, i.e., its non-membership function, obviously presents the preference not to select that particular cost. Altogether, the sum of these two preferences may be of the maximum value 1 . When selecting a route for transportation, the purchaser wishes to seek a way of delivering the goods that is associated with a minimum cost. In this case, the membership function takes a high value of preference and the non-membership function obviously takes a minimum value, because the seller always tries to give benefit to the purchaser for a strong future business relation. Meanwhile, due to some restrictions of vehicle types in different routes, the $D M$ cannot provide better policies to the customers in each business deal. Our investigation is established on the hesitant fuzzy approach to accommodate the given situation using the membership function according to the customers' point of view and the non-membership function for the sellers' point of view.
The transportation of goods is completed within different time schedules related to different routes or vehicles. If the transportation is made within a short period of time, then the cost of a unit commodity for transportation is larger than the cost of the case of a larger time of transportation. In this constellation, the purchaser creates two conflicting situations, namely, to minimize cost and to minimize time. However, in most of the cases, the buyer wants to minimize the transportation costs. Therefore, the route or vehicle associated with its minimum transportation cost is reflected by a membership function, and furthermore, there is a nonmembership function for minimizing the transportation time. Moreover, the buyer may give priority to transporting the goods as quickly as possible while not considering the transportation cost more effectively than the transportation time. According to our viewpoint, we would like to minimize the transportation cost with some important perspectives on the dimension of time. In fact, we do not introduce time directly in our model; instead, dual-hesitant fuzzy membership functions corresponding to different routes with different costs and times are introduced in the proposed DHFTP.

\section{Preliminaries}

Here, we tackle the uncertainty of TP based on the dualhesitant fuzzy numbers, and we recall the basic definitions and properties of HFSs and DHFSs.

\subsection{Hesitant fuzzy sets}

Earlier, the concept of a HFS was incorporated by Torra and Narukawa [18]. Thereafter, Torra [19] described it as presented subsequently. A HFS is usually defined in terms of a function that produces a set of membership values corresponding to each element in its domain. 
Definition 3.1 (Torra [19]). A HFS $H$ on $X$ is defined in terms of a function $h(x)$ that returns a subset of values in the interval $[0,1]$ once it is applied to $X$, i.e., an element of its power set:

$$
h: X \rightarrow \wp([0,1])
$$

Thereafter, $\mathrm{Xu}$ and Xia (2011) described the definition of HFS in a compact form by including the mathematical representation of a HFS.

Definition 3.2 (Xu and Xia [26]). A HFS is stated mathematically in the following way:

$$
H=\left\{\left(x_{i}, h\left(x_{i}\right)\right): x_{i} \in X\right\},
$$

where $h\left(x_{i}\right)$ is a set of several different values in the interval $[0,1]$ for each $x_{i} \in X$, which denotes the possible membership degree of the element $x_{i} \in X$ in the set $H$. In the usual sense, each member of $h\left(x_{i}\right)$ is called a Hesitant Fuzzy Element (HFE), denoted by $h_{i}$.

Definition 3.3 Suppose $h_{i}$ is a HFE of the HFS $H$ for some $x_{i} \in X$. Then its infimum and supremum are defined as $h_{i}^{-}=\inf _{x_{i} \in X} h\left(x_{i}\right)$ and $h_{i}^{+}=\sup _{x_{i} \in X} h\left(x_{i}\right)$, respectively.

Assumption Here, to accommodate multiple routes of transportation in our proposed study, we require the chosen set $X$ to be a finite and nonempty set. Consequently, in this paper, we address a finite and non-empty HFS without changing the original names such as HFS and HFE.

To make a difference between HFS and HFE, we define as follows: a HFS is a set of subsets in the interval $[0,1]$ and corresponds to a set for each element of the reference set $H$, whereas a HFE is one of such subsets.

\subsection{Score of a HFS}

Let us consider a HFS $H=\left\{\left(x_{i}, h\left(x_{i}\right)\right): x_{i} \in X\right\}$, where $X=$ $\left\{x_{1}, x_{2}, \ldots, x_{n}\right\}$ is a finite set. For a HFE $h_{i}$ in $H$, we define a score function, denoted as $s\left(h_{i}\right)$ and defined as follows:

$$
s\left(h_{i}\right)=\frac{\sum_{j=1}^{k} h\left(x_{j}\right)}{k}(i=1,2, \ldots, n) .
$$

Here, the real number $s\left(h_{i}\right)$ is called the score of $h_{i}$, where $k$ is the number of elements in $h_{i}$. Let $h_{1}$ and $h_{2}$ be two HFEs.

Case 3.2.1: $\quad$ If $s\left(h_{1}\right)>s\left(h_{2}\right)$, then $h_{1}$ is called superior to $h_{2}$, denoted by $h_{1}>h_{2}$.

Case 3.2.2: $\quad$ If $s\left(h_{1}\right)<s\left(h_{2}\right)$, then $h_{1}$ is called inferior to $h_{2}$, denoted by $h_{1}<h_{2}$.

Case 3.2.3: If $s\left(h_{1}\right)=s\left(h_{2}\right)$, then $h_{1}$ is called indifferent from $h_{2}$, denoted by $h_{1} \sim h_{2}$.

Here, we consider the aforementioned score function, which is chosen on the ground of our multi-choice study in TP; others may understand and apply a score function in another way, according to their choice in the respective field of research.

\subsection{Dual hesitant fuzzy sets}

Definition 3.4 Let $X$ be a fixed set; then a DHFS $D$ on $X$ is defined as follows:

$$
D=\{(x, h(x), g(x)): x \in X\}
$$

where $h(x)$ and $g(x)$ are mappings that take set-values in $[0,1]$; they are denoted as the possible membership degree and non-membership degree of any element $x \in X$ to the set $D$, respectively, with the conditions

$$
0 \leq h_{D}, g_{D} \leq 1,0 \leq h_{D}+g_{D} \leq 1,
$$

for any $h_{D} \in h(x), g_{D} \in g(x)$. A Dual-Hesitant Fuzzy Element (DHFE) is understood as the pair $d(x)=(h(x), g(x))$, and it is denoted in the functional form as $d=(h, g)$.

Consider any DHFE $d$; then it may fulfill the following criteria.

(i) Complete uncertainty: $d=\{\{0\},\{1\}\}$.

(ii) Complete certainty: $d=\{\{1\},\{0\}\}$.

(iii) Complete ill-knowledge (all is possible): $d=[0,1]$.

(iv) Nonsensical element: $d=\phi(h=\phi, g=\phi)$.

Here, under our perspective, which is given by a multichoice of roots in the TP, we consider $X$ as a finite set and, herewith, DHFS $D$ as finite; its elements are named as DHFE.

\subsection{Arithmetic operations on DHFEs}

Let $d_{1}=\left\{h_{d_{1}}, g_{d_{1}}\right\}$ and $d_{2}=\left\{h_{d_{2}}, g_{d_{2}}\right\}$ represent two DHFEs; then addition, subtraction and scalar multiplication of the numbers are stated as follows.

$$
\begin{aligned}
& \text { Addition } \\
& d_{1} \oplus d_{2}=\left\{h_{d_{1}} \oplus h_{d_{2}}, g_{d_{1}} \ominus g_{d_{2}}\right\}=\cup_{\gamma_{d_{1}} \in h_{d_{1}}, \eta_{d_{1}} \in g_{d_{1}}, \gamma_{d_{2}} \in h_{d_{2}}}, \\
& \eta_{d_{2}} \in g_{d_{2}}=\left\{\left\{\gamma_{d_{1}}+\gamma_{d_{2}}-\gamma_{d_{1}} \gamma_{d_{2}}\right\},\left\{\eta_{d_{1}} \eta_{d_{2}}\right\}\right\} . \\
& \text { Subtraction } \\
& d_{1} \ominus d_{2}=\left\{h_{d_{1}} \ominus h_{d_{2}}, g_{d_{1}} \oplus g_{d_{2}}\right\}=\cup_{\gamma_{d_{1}} \in h_{d_{1}}, \eta_{d_{1}} \in g_{d_{1}}, \gamma_{d_{2}} \in h_{d_{2}}}, \\
& \eta_{d_{2}} \in g_{d_{2}}=\left\{\left\{\gamma_{d_{1}} \gamma_{d_{2}}\right\},\left\{\eta_{d_{1}}+\eta_{d_{2}}-\eta_{d_{1}} \eta_{d_{2}}\right\}\right\} . \\
& \quad \text { Scalar } \text { multiplication: For any } \text { real } n, \\
& \text { nd }=\cup_{\gamma_{d} \in h_{d}, \eta_{d} \in g_{d}}=\left\{1-\left(1-\gamma_{d}\right)^{n},\left(\eta_{d}\right)^{n}\right\} . \\
& \text { If } n \text { is a positive integer then for any } n, \\
& d^{n}=\cup_{\gamma_{d} \in h_{d}, \eta_{d} \in g_{d}}=\left\{\left(\gamma_{d}\right)^{n}, 1-\left(1-\eta_{d}\right)^{n}\right\} .
\end{aligned}
$$

\subsection{Ranking of dual hesitant fuzzy sets}

Let $D=\{(x, h(x), g(x)): x \in X\}$ be a DHFS, where $X=$ $\left\{x_{1}, x_{2}, \ldots, x_{n}\right\}$ and $d=\left\{h_{d}, g_{d}\right\}$ be a DHFE. We define a score function $s_{d}$ on the DHFS, represented as follows: 


$$
s_{d}=\left|\frac{1}{k} \sum_{i=1}^{k} h_{d}\left(x_{i}\right)-\frac{1}{k} \sum_{i=1}^{k} g_{d}\left(x_{i}\right)\right| .
$$

Let $d_{1}$ and $d_{2}$ be any two DHFSs. With regard to a given score function, Zhu et al (2012) defined order relations as follows:

(i) if $s_{d_{1}}>s_{d_{2}}$, then $d_{1}$ is said to be superior to $d_{2}$, denoted by $d_{1}>d_{2}$;

(ii) if $s_{d_{1}}<s_{d_{2}}$, then $d_{1}$ is said to be inferior to $d_{2}$, denoted by $d_{1}<d_{2}$;

(iii) if $s_{d_{1}}=s_{d_{2}}$, then $d_{1}$ is said to be equivalent to $d_{2}$, denoted by $d_{1} \sim d_{2}$.

\section{Model formulation}

The mathematical formulation of a classical TP is given as follows:

$$
\begin{gathered}
\text { minimize } Z=\sum_{i=1}^{m} \sum_{j=1}^{n} c_{i j} x_{i j} \\
\text { subject to } \sum_{j=1}^{n} x_{i j} \leq a_{i}(i=1,2, \ldots, m), \\
\sum_{i=1}^{m} x_{i j} \geq b_{j}(j=1,2, \ldots, n), \\
x_{i j} \geq 0 \quad \forall i, j .
\end{gathered}
$$

Here, $Z$ stands for the objective function to manipulate total transportation cost; $c_{i j}(i=1,2, \ldots, m ; j=1,2, \ldots, n)$ is considered as transportation cost per unit commodity from $i$ th origin to $j$ th destination; $a_{i}(i=1,2, \ldots, m)$ and $b_{j}(j=$ $1,2, \ldots, n)$ are taken as availability and demand at $i$ th origin and $j$ th destination, respectively, and

$$
\sum_{i=1}^{m} a_{i} \geq \sum_{j=1}^{n} b_{j}
$$

is the feasibility condition.

The cost $c_{i j}$ is usually treated as being of deterministic nature. However, in real-life situations, the precise value of this transportation cost may not be known.

Studying in an uncertain environment of OR using HFS is well established and important in different real-life managerial fields like inventory optimization, supply chain management, etc. Considering the imprecise information on transportation parameters in TP, here, the HFS is incorporated to tackle some difficult situations that practically occur in a TP. Generally, the transportation cost is paid by the customer and he always needs to minimize the transportation cost. If there are several routes to supply the goods to the destinations, then the customer will select that route through which a minimum cost needs to be paid, but the supplier may not have the specific capacity to supply according to the purchasers' perspectives. Then a contradiction will occur when choosing the transportation route. However, it is true that the supplier also tries to supply the goods under minimum cost, and this restriction may create a high complexity to do so. In this situation, the HFS could be used to tackle the problem; the minimum cost of transportation refers to a high priority, which indicates that the membership value increases. Again, when the cost increases, then the priority decreases, which makes the nonmembership value to increase. Consider a route of transportation from $i$ th origin to the $j$ th destination in which the associated transportation cost is $c_{i j}$ per unit item of goods. However, there are some possibilities of selecting the respective route by purchaser and possibility to provide that particular route of transportation that is highly conflicting. Most of the customers select a route through which they pay minimum cost. In this situation, we cannot use the cost $c_{i j}$ directly for minimizing the transportation cost. Then we impose the idea of preference value $\left(h_{i j}\right)$ for purchaser corresponding to $c_{i j}$. It is certain that choosing minimum value of $c_{i j}$ is equivalent to selecting the high preference value of $h_{i j}$. Furthermore, the possibility of providing the routes with minimum cost is limited as they are highly preferred; hence, we introduce the possibility of not getting a preferred route by the value $g_{i j}$. Obviously, the purchasers would like to minimize the value $g_{i j}$ in the problem. Thus, corresponding to each route $t$, there are two values $h_{i j}^{t}$ and $g_{i j}^{t}$ (say) corresponding to $c_{i j}$. If $S$ be the set of all routes, we use the dual-hesitant fuzzy cost $\left\{\left(t, h_{i j}^{t}, g_{i j}^{t}\right): t \in S\right\}$. As $S$ contains different numbers of routes for different origins, we use the simplest form of notation for dual-hesitant fuzzy cost as $\tilde{c}_{i j}=\left(h_{i j}, g_{i j}\right)$ in the rest of our discussion. Hence, the dual-hesitant fuzzy cost $\left(\tilde{c}_{i j}\right)$ is introduced in order to design the mathematical model of the TP; we name it as a $D H F T P R$ and we put this in the following way:

$$
\text { optimize } z=\sum_{i=1}^{m} \sum_{j=1}^{n} \tilde{c}_{i j} x_{i j}
$$

subject to the constraints (7)-(9).

In fact, the objective function $(z)$ in problem (11) is designed based on dual-hesitant fuzzy costs $\tilde{c}_{i j}$. In accordance with the real situation, we assign aspiration values in favour of selecting a route (membership values), and another kind in opposition to the selection of this route (non-membership values). Here, we regard the membership and non-membership values of dual-hesitant fuzzy cost as $\left(h_{i j}, g_{i j}\right)$, where $h_{i j}$ and $g_{i j}$ are the membership value and the non-membership value, respectively, which are connected with a transportation cost, corresponding to a route. In this situation, the DMs wish to minimize the total transportation cost; hence they need to maximize the membership value 
$h_{i j}$ (as it is favourable by the DM) and to minimize the nonmembership value $g_{i j}$, in order to find an optimal solution of the optimization problem (11). Based on these reflections, the optimum solution of the program (11) is equivalent to the optimum solution of the optimization problem specified as follows:

$$
\text { optimize } z=\sum_{i=1}^{m} \sum_{j=1}^{n}\left(h_{i j}, g_{i j}\right) x_{i j}
$$

subject to the constraints $(7)-(9)$.

Here, the two parameters $h_{i j}$ and $g_{i j}$ are related in such a way that if the cost is minimal, then $h_{i j}$ takes a larger value, whereas $g_{i j}$ is chosen with a smaller value for the corresponding minimum cost at the node. We maximize the acceptance degrees $h_{i j}$ and minimize the rejection degrees $g_{i j}$ in the sense of problem (12). Hence, the objective function of our problem reduces to two objective functions. They are defined in the following bi-objective problem formulation:

$$
\begin{aligned}
& \text { maximize } z=\sum_{i=1}^{m} \sum_{j=1}^{n} h_{i j} x_{i j} \\
& \text { minimize } z=\sum_{i=1}^{m} \sum_{j=1}^{n} g_{i j} x_{i j} \\
& \text { subject to the constraints }(7)-(9) .
\end{aligned}
$$

Here, we assume that the values of the functions on membership and non-membership are less than 1; this is represented as constraints in the proposed model. Thus, the DHFTPR becomes a linear programming problem with two objective functions and it is stated as follows:

$$
\begin{aligned}
& \operatorname{maximize} z=\sum_{i=1}^{m} \sum_{j=1}^{n} h_{i j} x_{i j} \\
& \text { minimize } z=\sum_{i=1}^{m} \sum_{j=1}^{n} g_{i j} x_{i j}
\end{aligned}
$$

subject to $\left(h_{i j}+g_{i j}-1\right) x_{i j} \leq 0$

$(i=1,2, \ldots, m ; j=1,2, \ldots, n)$

and the constraints $(7)-(9)$.

\section{Solution procedure of DHFTPR by VAM}

Now, we discuss the solution procedure of a DHFTPR by introducing the following steps with the help of VAM (cf. Reinfeld and Vogel [27]).

- Step 1: Considering the priorities of the routes and the possibilities of supplying items through the routes, we construct a dual-hesitant fuzzy cost function $\tilde{c}_{i j}$ and convert it into a crisp cost using a score function.

- Step 2: Determine the difference between the lowest costs of two cells in all rows and columns, including dummy cell, if any and write them at the bottom of the Dual-Hesitant Fuzzy Transportation Table (DHFTT) under the corresponding columns.

- Step 3: Allocate as much as possible to the lowest-cost cell in the row or column with the highest difference. If two or more differences are equal, allocate as most possible to the lowest-cost cell in these rows or columns.

- Step 4: Stop the process if all rows and columns requirement are met. If not, go to the next step.

- Step 5: Calculate the difference between the two lowest cells remaining in all rows and columns. Any row and column with zero supply or demand should not be used in calculating further differences. Then go to Step 2.

- Step 6: The Initial Dual-Hesitant Fuzzy Basic Feasible Solution (IDHFBFS) and the initial dual-hesitant fuzzy transportation cost are $x_{i j}$ and $\sum_{i=1}^{m} \sum_{j=1}^{n} c_{i j} x_{i j}$, respectively.

\subsection{Generalized dual-hesitant fuzzy modified distribution method}

Here, the Generalized Dual-Hesitant Fuzzy Modified Distribution Method (GDHFMDM) is introduced to find the dual hesitant fuzzy optimal solution of the DHFTP with the help of Initial Basic Feasible Solution (IBFS).

The following steps are conducted to find the dual-hesitant fuzzy optimal solution:

- Step 1: Find the IDHFBFS of DHFTP using VAM.

- Step 2: Introduce the dual-hesitant fuzzy dual variables $u_{i}$ and $v_{j}$ corresponding to each $i$-th row and $j$-th column, respectively. Write $u_{i}$ in front of each $i$-th row and $v_{j}$ at the bottom of each $j$-th column. Take any one of $u_{i}$ or $v_{j}$ to be zero, where the number of cell allocations is maximal.

- Step 3: For basic cells, calculate $u_{i}+v_{j}=c_{i j}$ for all $i$ and $j$. This relation assigns the values to all $u_{i}$ and $v_{j}$.

- Step 4: For non-basic cells find the score of $d_{i j}=$ $c_{i j}-\left(u_{i}+v_{j}\right)$ and write it on the left side of the concerned cell. The following two cases may arise:

Case 4.1: If $d_{i j} \geq 0 \quad \forall i, j$, then the obtained IDHFBFS is a fuzzy optimal solution.

Case 4.2: If there exists at least one $d_{i j}$ such that $d_{i j}<0$, then the obtained IDHFBFS is not an optimal solution. Go to Step 5.

- Step 5: In the IDHFBFS, choose $d_{i j}$ whose score value is the most negative. Let it be at $(r, s)$ cell, where $1 \leq r \leq m$ and $1 \leq s \leq n$. 
- Step 6: Assign a positive quantity $\theta$ in the $(r, s)$ cell corresponding to which the score value of $d_{i j}$ is the most negative, and make a loop as follows.

Rule defining the loop: Start from the $\theta$ cell and move horizontally and vertically to the nearest basic cell with the restriction that the full turn of the loop must not lie in any non-basic cell (except for the $\theta$ cell). In this way, we return to the $\theta$ cell in order to complete the loop.

- Step 7: Start from the $(r, s)$ cell; allocate the amount $\theta$ with alternating positive and negative signs to all the end points of the closed loop, so that supply and demand constraints are always satisfied. The value of $\theta$ is chosen as the minimum quantity that will render non-negative values for all the basic variables in the new solution.

- Step 8: Insert the fixed value of $\theta$ in the cell, where the next DHFBFS is obtained; this improves the initial fuzzy transportation cost. While inserting the value of $\theta$, one cell assumes the 0 value. Here, 0 will not be mentioned as this is the fuzzy leaving variable, i.e., this cell becomes non-basic. This yields an improved DHFBFS.

- Step 9: Again, use the latest DHFBFS, and repeat Steps 1-8 until $d_{i j} \geq 0 \quad \forall i, j$.

- Step 10: The obtained dual-hesitant fuzzy optimal solution and fuzzy optimal cost are $x_{i j}$ and $\sum_{i=1}^{m} \Sigma_{j=1}^{n} c_{i j} \cdot x_{i j}$, respectively.

Remark 1 Solving a TP by VAM, the cells where the allocations are made, i.e., where $x_{i j}>0$, are called basic cells, and the cells where the allocations are not assigned, i.e., where $x_{i j}=0$, are treated as non-basic cells (cf. Bertsimas and Tsitsiklis [28]).

\section{Numerical example}

A reputed company has three sugar factories $S_{1}, S_{2}$ and $S_{3}$, and the sugar sorts are distributed into three markets $D_{1}, D_{2}$ and $\mathrm{D}_{3}$, respectively. There are different types of vehicles to transport sugar to the destinations. Corresponding to each type of vehicles, different transportation costs are assigned. During sugar supply, it is noticed that dealers always give more preference to a choice of vehicles with a minimum cost, but it is not possible for the supplier to maintain in their supplying system. Hence, there are some bounded capacities of the company regarding the vehicle for transporting the sugar; this causes some non-preferable situations in the problem. Consequently, the hesitant fuzzy situation for selecting the vehicles is chosen. Hence, the main aim of the company is to minimize the total transportation cost through a TP under a dual-hesitant fuzzy environment.

Table 2 provides the availability $a_{i}(i=1,2,3)$ of the sugar at three sources $\mathrm{S}_{i}(i=1,2,3)$, the demands $b_{j}(j=$
$1,2,3)$ at three destinations $\mathrm{D}_{j}(j=1,2,3)$ and the approximate cost for transporting one unit of sugar $(10 \mathrm{~kg})$ from any source to any destination is represented by dualhesitant fuzzy numbers. We determine the DHFTP and obtain the optimal solution. According to our proposed algorithm, at first we need to find the score values along with the associated ranking values corresponding to the hesitant fuzzy costs. The score values and the fuzzy cost ranking values are shown in table 3. To find the optimal solution of the problem, we use the procedure of VAM, which is presented in section 4 . The iteration at each step of the allocation is provided in tables 4, 5, 6, 7, 8, 9, and an optimality test is shown in table 10 .

\section{Cell allocation by VAM}

Now we see from table 10 that

$$
\begin{aligned}
& d_{11}=c_{11}-\left(u_{1}+v_{1}\right)=0.27-(0.3-0.03)=0, \\
& d_{13}=c_{13}-\left(u_{1}+v_{3}\right)=0.07-(-0.03+0.025)=0.075 \\
& d_{23}=c_{23}-\left(u_{2}+v_{3}\right)=0.14-(-0.005+0.025)=0.12 \\
& d_{32}=c_{32}-\left(u_{3}+v_{2}\right)=0.55-(0+0.205)=0.345
\end{aligned}
$$

Since $d_{i j} \geq 0$ for all $i$ and $j$, the optimal solution is $x_{12}=$ $20, x_{21}=20, x_{22}=4, x_{31}=15, x_{33}=20$ and the transportation cost has the value

$$
\begin{aligned}
& 20 \cdot 21.25+20 \cdot 13.5+4 \cdot 35+15 \cdot 38.33+20 \cdot 39.25 \\
& \quad=2194.95 .
\end{aligned}
$$

\section{Comparison to existing methods}

The TP is one of the utmost important decision-making problems that occur in daily-life OR situations. From the classical viewpoint of TP, its model is used to minimize the total transportation cost of the transportation system while satisfying supply and demand restrictions. In most of the cases, the transportation cost is paid by the buyer. Therefore, the model of the classical TP is helpful for buyers, but it does not give any priority to sellers as well as to the supplying capacity. As a whole, the mathematical model of TP has the capability only to minimize transportation cost without considering any other important aspects and factors. However, in many research works (cf. Chanas and Kuchta [14], Kaur and Kumar [15], Ebrahimnejad [29]), fuzzy parametric situations are presented with some important factors for various decision-making problems, but they also consider the particular minimizing sense, which is favourable for the buyers. However, in reality, it is not always possible to supply the goods satisfying the buyers' criterion for the sellers' due to shortages of proper vehicles along different routes. In those situations, either the customers need to pay a higher cost for getting the ordered quantity in a supplementary way within a proper time, or they would wait for getting the ordered quantity 
Table 2. Formulating dual-hesitant fuzzy cost.

\begin{tabular}{|c|c|c|c|c|}
\hline & $\mathrm{D}_{1}$ & $\mathrm{D}_{2}$ & $\mathrm{D}_{3}$ & $a_{i}$ \\
\hline $\mathrm{S}_{1}$ & $\begin{array}{c}\{\{0.5,0.4,0.1\},\{0.4,0.5,0.9\}\} \\
(20,25,30)\end{array}$ & $\begin{array}{c}\{\{0.7,0.6,0.5,0.2\},\{0.1,0.3,0.4,0.5\}\} \\
(14,16,20,35)\end{array}$ & $\begin{array}{c}\{\{0.6,0.4,0.3\},\{0.2,0.6,0.7\}\} \\
(22,25,36)\end{array}$ & 20 \\
\hline $\mathrm{S}_{2}$ & $\begin{array}{c}\{\{0.4,0.2\},\{0.3,0.5\}\} \\
(12,15)\end{array}$ & $\begin{array}{c}\{\{0.7,0.6,0.3\},\{0.1,0.3,0.6\}\} \\
(30,35,40)\end{array}$ & $\begin{array}{c}\{\{0.6,0.5,0.3\},\{0.2,0.3,0.5\}\} \\
(22,27,30)\end{array}$ & 24 \\
\hline $\mathrm{S}_{3}$ & $\begin{array}{c}\{\{0.3,0.2,0.1\},\{0.2,0.6,0.7\}\} \\
(30,40,45)\end{array}$ & 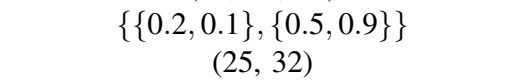 & $\begin{array}{c}\{\{0.6,0.5,0.3,0.2\},\{0.2,0.3,0.5,0.7\}\} \\
(32,35,40,50)\end{array}$ & 35 \\
\hline$b_{j}$ & 35 & 24 & 20 & \\
\hline
\end{tabular}

Table 3. Score value and fuzzy cost ranking value.

\begin{tabular}{ccccc}
\hline & $\mathrm{D}_{1}$ & $\mathrm{D}_{2}$ & $\mathrm{D}_{3}$ & $a_{i}$ \\
\hline $\mathrm{S}_{1}$ & $0.27 / 25$ & $0.175 /$ & $0.07 / 27.67$ & 20 \\
& & 21.25 & & \\
$\mathrm{~S}_{2}$ & $0.1 / 13.5$ & $0.2 / 35$ & $0.14 / 26.33$ & 24 \\
$\mathrm{~S}_{3}$ & $0.3 /$ & $0.55 / 28.5$ & $0.025 /$ & 35 \\
& 38.33 & & 39.25 & \\
$b_{j}$ & 35 & 24 & 20 & \\
\hline
\end{tabular}

Table 4. First iteration of cost matrix by VAM.

\begin{tabular}{lcccc}
\hline & $\mathrm{D}_{1}$ & $\mathrm{D}_{2}$ & $\mathrm{D}_{3}$ & $a_{i}$ \\
\hline $\mathrm{S}_{1}$ & 0.27 & 0.175 & 0.07 & $20(0.105)$ \\
$\mathrm{S}_{2}$ & 0.1 & 0.2 & 0.14 & $24(0.04)$ \\
$\mathrm{S}_{3}$ & 0.3 & 0.55 & 0.025 & $35(0.275)$ \\
$b_{j}$ & $35(0.17)$ & $24(0.025)$ & $20(0.07)$ & \\
\hline
\end{tabular}

Table 5. Second iteration of cost matrix by VAM.

\begin{tabular}{lcccc}
\hline & $\mathrm{D}_{1}$ & $\mathrm{D}_{2}$ & 3 & $a_{i}$ \\
\hline $\mathrm{S}_{1}$ & 0.27 & 0.175 & 0.07 & $20(0.095)$ \\
& & & - & \\
$\mathrm{S}_{2}$ & 0.1 & 0.2 & 0.14 & $24(0.1)$ \\
$\mathrm{S}_{3}$ & 0.3 & 0.55 & 0.025 & $15(0.25)$ \\
& & & 20 & \\
$b_{j}$ & $35(0.17)$ & $24(0.025)$ & - & \\
\hline
\end{tabular}

with a time delay. According to the mentioned situations, sometimes a customer pays a larger cost to purchase goods within time; otherwise, he or she considers a late transport according to the given vehicle capacity for paying less. Under the discussed conditions, it is almost impossible to tackle the problem by the well-known methods. Because of
Table 6. Third iteration of cost matrix by VAM.

\begin{tabular}{lcccc}
\hline & $\mathrm{D}_{1}$ & $\mathrm{D}_{2}$ & $\mathrm{D}_{3}$ & $a_{i}$ \\
\hline $\mathrm{S}_{1}$ & 0.27 & 0.175 & 0.07 & $20(0.095)$ \\
& & & - & \\
$\mathrm{S}_{2}$ & 0.1 & 0.2 & 0.14 & $24(0.1)$ \\
& & & - & \\
$\mathrm{S}_{3}$ & 0.3 & 0.55 & 0.025 & - \\
& 15 & - & 20 & \\
$b_{j}$ & $20(0.17)$ & $24(0.025)$ & - & \\
\hline
\end{tabular}

Table 7. Fourth iteration of cost matrix by VAM.

\begin{tabular}{ccccc}
\hline & $\mathrm{D}_{1}$ & $\mathrm{D}_{2}$ & $\mathrm{D}_{3}$ & $a_{i}$ \\
\hline $\mathrm{S}_{1}$ & 0.27 & 0.175 & 0.07 & $20(0.175)$ \\
& - & & - & \\
$\mathrm{S}_{2}$ & 0.1 & 0.2 & 0.14 & $4(0.2)$ \\
& 20 & & - & \\
$\mathrm{S}_{3}$ & 0.3 & 0.55 & 0.025 & - \\
& 15 & - & 20 & \\
$b_{j}$ & - & $24(0.025)$ & - & \\
\hline
\end{tabular}

Table 8. Fifth iteration of cost matrix by VAM.

\begin{tabular}{ccccc}
\hline & $\mathrm{D}_{1}$ & $\mathrm{D}_{2}$ & $\mathrm{D}_{3}$ & $a_{i}$ \\
\hline $\mathrm{S}_{1}$ & 0.27 & 0.175 & 0.07 & $-(0.175)$ \\
& - & 20 & - & \\
$\mathrm{S}_{2}$ & 0.1 & 0.2 & 0.14 & $-(0.2)$ \\
& 20 & 4 & - & \\
$\mathrm{S}_{3}$ & 0.3 & 0.55 & 0.025 & - \\
& 15 & - & 20 & \\
$b_{j}$ & - & $24(0.025)$ & - & \\
\hline
\end{tabular}

this fact, we introduce our proposed approach using a dualhesitant fuzzy environment.

In our suggested methodology, we allow for priority to consider both the buyers' and the sellers' perspectives. The 
Table 9. Sixth iteration of cost matrix by VAM.

\begin{tabular}{lcccc}
\hline & $\mathrm{D}_{1}$ & $\mathrm{D}_{2}$ & $\mathrm{D}_{3}$ & $a_{i}$ \\
\hline $\mathrm{S}_{1}$ & 0.27 & 0.175 & 0.07 & 24 \\
& & 20 & & \\
$\mathrm{~S}_{2}$ & 0.1 & 0.2 & 0.14 & 35 \\
& 20 & 4 & & \\
$\mathrm{~S}_{3}$ & 0.3 & 0.55 & 0.025 & 35 \\
& 15 & & 20 & \\
$b_{j}$ & 35 & 24 & 20 & \\
\hline
\end{tabular}

Table 10. Optimality test of TP.

\begin{tabular}{lcccc}
\hline & $\mathrm{D}_{1}$ & $\mathrm{D}_{2}$ & $\mathrm{D}_{3}$ & $u_{i}$ \\
\hline $\mathrm{S}_{1}$ & 0.27 & 0.175 & 0.07 & -0.03 \\
& 0 & 20 & 0.075 & \\
$\mathrm{~S}_{2}$ & 0.1 & 0.2 & 0.14 & -0.005 \\
& 20 & 4 & 0.12 & \\
$\mathrm{~S}_{3}$ & 0.3 & 0.55 & 0.025 & 0 \\
& 15 & 0.345 & 20 & \\
$v_{j}$ & 0.3 & 0.205 & 0.025 & \\
\hline
\end{tabular}

Table 11. TP with minimum cost.

\begin{tabular}{llll}
\hline & $\mathrm{D}_{1}$ & $\mathrm{D}_{2}$ & $\mathrm{D}_{3}$ \\
\hline $\mathrm{S}_{1}$ & 20 & 14 & 22 \\
$\mathrm{~S}_{2}$ & 12 & 30 & 22 \\
$\mathrm{~S}_{3}$ & 30 & 25 & 32 \\
\hline
\end{tabular}

presented numerical example includes parameters with a HFS. We see that there are maximum and minimum transportation costs at each of the nodes. Among these, the minimum transportation costs are more preferable for customers than the maximum transportation costs. Due to this fact, a hesitant membership function is formulated in our study. To justify the better impact of our study in comparison with the traditional approaches, we first consider minimum cost at each of the cells; then we find the cost matrix, which is given in table 11 .

Applying VAM, we formulate and solve the TP involving the cost parameters of table 11 ; the feasibility restriction is described in our numerical example. Thereafter, we derive the solution as follows:

$x_{12}=20, x_{21}=24, x_{22}=11, x_{32}=4, x_{33}=20$ and the transportation cost is 1638 .

This solution is preferred by the buyers, as in this case, the transportation cost is minimized along each of the routes for transporting the goods.

Again, if we consider the maximum cost at each of the cells, we find the cost matrix that is given in table 12 .
Table 12. TP with maximum cost.

\begin{tabular}{llll}
\hline & $\mathrm{D}_{1}$ & $\mathrm{D}_{2}$ & $\mathrm{D}_{3}$ \\
\hline $\mathrm{S}_{1}$ & 30 & 35 & 36 \\
$\mathrm{~S}_{2}$ & 15 & 40 & 30 \\
$\mathrm{~S}_{3}$ & 45 & 32 & 50 \\
\hline
\end{tabular}

Applying VAM, we formulate and solve the TP using the cost parameters from table 12 under the feasibility restriction as provided in our numerical example. Then, we get the following solution:

$x_{12}=11, x_{13}=9, x_{21}=15, x_{32}=24, x_{33}=11$ and the transportation cost is 2332 . This solution is preferred by the sellers, as in this case, the capacity of supplying goods for the sellers' side is easy, since the vehicles are available frequently for transporting the goods. Thus, from this discussion we observe that the two solutions are very much conflicting as well as biased.

With these results, we see that transportation cost (with minimum HFS cost) $<$ transportation cost (with score value) $<$ transportation cost (with maximum HFS cost);

indeed, $1638<2194.95<2332$.

Based on these relations, we realize that the solution obtained with our approach lies is both the best appreciated solution by the buyer and by the seller. Herewith, we have arrived at a solution, which provides a minimization of the transportation cost, not only from the customers' point of view but also from the sellers' perspective. Thus, the solution by our approach can be interpreted as unbiased.

Based on our proposed approach, many bi-objective decision problems (cf. Salazar et al [30]) that occur in practically relevant situations can be handled by our methodology. Most convincingly, if the objective functions are of conflicting nature with one of maximizing and another one of minimizing type, then we can find a better compromise solution by incorporating membership and non-membership functions, corresponding to them, respectively. Hence, the regarded bi-objective problem can be solved by our algorithm using VAM without any computational burden. In this regard, we relate our approach also to solve the problem investigated by Ehrgott et al [31]. In fact, the bi-objective route selection problem of that work can be treated through our newly introduced algorithm, using a DHFS based on our proposition of membership and non-membership functions in the objective function. Hence, our study on DHFTP may be helpful and valuable in order to find an optimal solution in numerous areas of daily-life uncertain decision-making problems.

\section{Conclusion and outlook}

In the area of optimization, a large number of TPs in different areas have been studied. However, some of the problems have a limited scope of application in real-life 
situations due to the need for the presence of crisp data of transportation parameters, such as cost, supply and demand, in the formulated model. Furthermore, there is a number of research works on uncertain TPs accommodating real-life decision-making problems using various uncertainty representations and, among them, fuzzy uncertainty. Contrary to traditional TPs under fuzziness, in our approach, we introduced a new class of uncertain TPs, namely, DHFTPs. The situation of a DHFTP was illustrated by us and its mathematical model was formulated. Algorithms were designed and described to find the optimal solution of DHFTP. A numerical example was provided to justify the proposed algorithms as well as the underlying model, and their utility in real-life decision-making problems was demonstrated. We especially provided research directions for associating with the method by Ehrgott et al [31] on scheduling vehicle routes in an urban city.

Various further research directions can be opened, based on our proposed methodology. According to the discussion in section 6, a delay time of transportation could be added to our model for getting more realistic results. One might initiate a two-fold uncertain environment such as hesitant fuzzy-stochastic, hesitant fuzzy-rough, etc., with our study as a foundation. Another new contribution may refer to different kinds of uncertainty, so-called hesitant intuitionistic fuzzy sets, for our new model and algorithms. This will serve in tackling challenges of information uncertainty within decision-making problems. We must emphasize that in connection to this paper, there is a line of future research directions to include the fixed-charge TP (cf. [32, 33]) under dual-hesitant fuzzy environment and that we have not raised it because it is outside the objectives initially set. Interested readers can formulate the fixed-charge transportation model under dual-hesitant fuzzy environment and solve it by the classical approach or any other suitable approach. In addition to all the aforementioned, we can employ our approach to find a compromise solution in inventory optimization, supply chain management and other emerging areas of OR and Economics.

\section{Acknowledgements}

Gurupada Maity is very thankful to the University Grants Commission of India for providing financial support to continue this research work under JRF(UGC) scheme: Sanction letter number [F.17-130/1998(SA-I)] dated 26/06/2014. The research of Sankar Kumar Roy is partially supported by the Portuguese Foundation for Science and Technology ("FCT-Fundação para a Ciência e a Tecnologia"), through the CIDMA-Center for Research and Development in Mathematics and Applications, University of Aveiro, Portugal, within the project UID/MAT/04106/2019. The research of Gerhard-Wilhelm Weber is partially supported by the Portuguese Foundation for Science and Technology ("FCT-Fundação para a
Ciência e a Tecnologia"), through the CIDMA-Center for Research and Development in Mathematics and Applications, University of Aveiro, Portugal. The authors are very much thankful to the Corresponding Editor and anonymous reviewers for their precious comments that helped them very much to rigorously improve the quality of the paper.

\section{References}

[1] Hitchcock F L 1941 The distribution of a product from several sources to numerous localities. Journal of Mathematics and Physics 20(1): 224-230

[2] Koopmans T C 1949 Optimum utilization of the transportation system. Econometrica 17: 136-146

[3] Kantorovich L V 1960 Mathematical methods of organizing and planning production. Management Science 6(4): 366-422

[4] Chanas S, Kolodziejczyk W and Machaj A 1984 A fuzzy approach to the transportation problem. Fuzzy Sets and Systems 13(3): 211-221

[5] Mahapatra D R, Roy S K and Biswal M P 2013 Multi-choice stochastic transportation problem involving extreme value distribution. Applied Mathematical Modelling 37(4): $2230-2240$

[6] Maity G and Roy S K 2016 Solving a multi-objective transportation problem with nonlinear cost and multi-choice demand. International Journal of Management Science and Engineering Management 11(1): 62-70

[7] Maity G, Roy S K and Verdegay J L 2016 Multi-objective transportation problem with cost reliability under uncertain environment. International Journal of Computational Intelligence Systems 9(5): 839-849

[8] Maiyar L M and Thakkar J J 2017 A combined tactical and operational deterministic food grain transportation model: particle swarm based optimization approach. Computers \& Industrial Engineering 110: 30-42

[9] Mogale D G, Kumar S K and Tiwari M K 2016 Two-stage Indian food grain supply chain network transportation-allocation model. IFAC-PapersOnLine 49(12): 1767-1772

[10] Roy S K and Maity G 2017 Minimizing cost and time through single objective function in multi-choice interval valued transportation problem. Journal of Intelligent and Fuzzy Systems 32: 1697-1709

[11] Rani D and Gulati T R 2016 Uncertain multi-objective multiproduct solid transportation problems Sadhana 41(5): 531-539

[12] Roy S K, Maity G, Weber G W and Alparslan Gök S Z 2017 Conic scalarization approach to solve multi-choice multiobjective transportation problem with interval goal. Annals of Operations Research 253(1): 599-620

[13] Roy S K, Maity G and Weber G W 2017 Multi-objective two-stage grey transportation problem using utility function with goals. Central European Journal of Operations Research 25: 417-439

[14] Chanas S and Kuchta D 1996 A concept of the optimal solution of the transportation problem with fuzzy cost coefficients. Fuzzy Sets and Systems 82(3): 299-305 
[15] Kaur A and Kumar A 2012 A new approach for solving fuzzy transportation problems using generalized trapezoidal fuzzy numbers. Applied Soft Computing 12(3): 1201-1213

[16] Jiménez F and Verdegay J L 1999 Solving fuzzy solid transportation problems by an evolutionary algorithm based parametric approach. European Journal of Operational Research 117(3): 485-510

[17] Roy S K, Ebrahimnejad A, Verdegay J L and Das S 2018 New approach for solving intuitionistic fuzzy multi-objective transportation problem. Sadhana 43(1). https://doi.org/10. 1007/s12046-017-0777-7

[18] Torra V and Narukawa Y 2009 On hesitant fuzzy sets and decision. In: Proceedings of the IEEE International Conference on Fuzzy Systems. https://doi.org/10.1109/FUZZY. 2009.5276884, pp. 1378-1382

[19] Torra V 2010 Hesitant fuzzy sets. International Journal of Intelligent Systems 25(6): 529-539

[20] Zhu B, Xu Z and Xia M 2012 Dual hesitant fuzzy sets. Journal of Applied Mathematics. https://doi.org/10.1155/ 2012/879629

[21] Singh P 2014 A new method for solving dual hesitant fuzzy assignment problems with restrictions based on similarity measure. Applied Soft Computing 24: 559-571

[22] Maity G and Roy S K 2017 Multi-objective transportation problem using fuzzy decision variable through multi-choice programming. International Journal of Operations Research and Information Systems 8(3): 82-96

[23] Mogale D G, Kumar M, Kumar S K and Tiwari M K 2018 Grain silo location-allocation problem with dwell time for optimization of food grain supply chain network. Transportation Research Part E: Logistics and Transportation Review 111: 40-69

[24] Mogale D G, Kumar S K and Tiwari M K 2018 An MINLP model to support the movement and storage decisions of the
Indian food grain supply chain. Control Engineering Practice 70: 98-113

[25] Mogale D G, Dolgui A, Kandhway R, Kumar S K and Tiwari M K 2017 A multi-period inventory transportation model for tactical planning of food grain supply chain. Computers \& Industrial Engineering 110: 379-394

[26] Xu Z and Xia M 2011 Distance and similarity measures for hesitant fuzzy sets. Information Sciences 181(11): 2128-2138

[27] Reinfeld N Y and Vogel W R 1958 Mathematical programming. Englewood Cliffs, New Jersey: Prentice-Hall

[28] Bertsimas D and Tsitsiklis J N 1997 Introduction to linear optimization. Belmont, Mass.: Athena Scientific. ISBN 978-1-886529-19-9

[29] Ebrahimnejad A 2014 A simplified new approach for solving fuzzy transportation problems with generalized trapezoidal fuzzy numbers. Applied Soft Computing 19: 171-176

[30] Salazar I A M, Molina J, Bello F A, Gómez T and Caballero R 2014 Solving a bi-objective transportation location routing problem by metaheuristic algorithms. European Journal of Operational Research 234(1): 25-36

[31] Ehrgott M, Wang J Y T, Raith A and Houtte C V 2012 A biobjective cyclist route choice model. Transportation Research Part A: Policy and Practice 46(4): 652-663

[32] Midya S and Roy S K 2014 Single-sink, fixed-charge, multiobjective, multi-index stochastic transportation problem. American Journal of Mathematical and Management Sciences 33: 300-314

[33] Midya S and Roy S K 2017 Analysis of interval programming in different environments and its application to fixedcharge transportation problem. Discrete Mathematics Algorithms and Applications 9(3). https://doi.org/10.1142/ S1793830917500409 